\title{
Ancora su Lope e Marino
}

\section{Paolo Cherchi}

Il capitolo dei debiti di Marino per Lope de Vega è il piú vecchio nella storia di questo tipo di ricerca riguardante il poeta napoletano. La Lira era appena apparsa (1614) che gia Alessandro Castelvestri, parlando per Fulvio Testi alle cui Rime (1617) scriveva la prefazione alludeva ai furti del Marino da poeti spagnoli; ${ }^{1}$ e non era una malignità, che nella terza parte della Lira Marino spacciava come suoi una buona dozzina di sonetti tradotti da Lope. L'accusa fu ripetuta infinite volte nelle polemiche fra marinisti e antimarinisti; ma il numero di componimenti plagiati e quali essi fossero precisamente fu per la prima volta stabilito da Meninni (161). Le ricerche piú vicine a noi, ${ }^{2}$ oltre a puntualizzare con nuovi dati, e in qualche caso anche con espunzioni, la portata del debito mariniano, ha potuto accertare che non solo la Lira ma anche La Galleria devono molto alle Rimas di Lope nonché all'Arcadia dello stesso. Il conto è questo: 16 sonetti (14 dalle Rimas del 1604 e 2 dall'Arcadia, 30 epigrammi (14 dalle Rimas e 16 dall'Arcadia) e, infine, la canzonetta "Sopra il ritratto della sua Donna A Domenico Pasignano" ("O memoria gentile") viene ripreso da "Anfrisio al retrato" dell'Arcadia. Un bottino ingente! Ma quest'opera rapace non avrebbe contaminato l'Adone, il capolavoro, considerando che il nome di Lope non è mai invocato fra quelli le cui opere subirono una notevole potatura dalla "ronchetta" mariniana. O meglio: il nome di Lope è stato fatto da J. Fucilla (Fucilla, "A classical theme" 289-90) per l'episodio dell'ape e Cupido (canto VI, 188-92) che avrebbe la sua fonte nella commedia Venus y Adonis (II.10) di Lope; ma D. Alonso (103-105) ha provato susseguentemente che la fonte di Marino sia un romance del Romancero General e non la versione che Lope ne diede nella sua commedia. Mi pare, tuttavia, che la tentazione di rifarsi ad un autore ammirato come Lope, perduri anche nell'Adone, e se ne vuol dare qui una prova.

Il passo mariniano in questione è l'iconologia della Gelosia, il motore che, al canto XII, spinge in una direzione affatto nuova la narrazione del poema. Nella descrizione di questo mostro, Marino prelevò delle tessere dal romance "Leriano a los celos" del secondo libro dell'Arcadia di Lope, opera, come abbiamo visto, frequentatissima da Marino. I prelievi si addensano in due ottave ma senza rispettare l'ordine del modello, per cui non è possibile ricorrere all'espediente delle due colonne che consente 
raffronti immediati: bisognerà perciò procedere immagine per immagine.

L'avvio del ritratto di "antica donna" (ott. 15) utilizza, come ha notato Giovanni Pozzi, attributi che l'antichità dava alle Furie. L'ottava 17 cambia registro:

Come Giano ha duo volti ed apre e gira

cento lumi qual Argo e piangon tutti

Di cervo il capo e la natura e l'atto

che si rivolge indietro a tratto a tratto.

Per Lope Gelosia ha "dos caras como el engaño / una humana, otra divina," e "la cabeza de ciervo."

Ecco quindi l'ottava 18, franta in 4 gruppi di due versi ciascuno:

Tolse le parolette alle fè greca,

la lingua mentitrice ala bugia

corrisponde a Lope

Los ojos hurtó a la ira;

los deseos, a los ciegos;

la fe y palabra, a los griegos; ,

y la lengua a la mentira.

E ancora

È il suo veder, come veder di cieca,

un vano imaginar di fantasia

che riprende

y es como vista di ciego

que está en la imaginación

Continua Marino:

Tende l'orecchie a chi novelle arreca

ed ha piè di ladron, passi di spia

che rifanno:

Tiene los pasos de espia

Tiene los pies de ladrón.

Conclude l'ottava:

D'alchimista il color pallido e mesto

e i dolori del parto in ogni gesto

L'associazione di gelosia e alchimia appare in Lope in un contesto leggermente diverso:

La color tiene de cuervo

y como dragón la vista,

las quimeras de alquimista 
y la cabeza de ciervo.

mentre l'immagine della partoriente è ripresa con maggior fedeltà:

Trae como ciego el tiento,

jamás de preguntas harto;

y como mujer de parto

las quejas y el movimiento.

Nelle ottave che seguono (19-22) appaiono temi che figurano anche in Lope: insonnia, tristezza e macchinazioni di Gelosia che si risolvono in suo danno. Tuttavia la mancanza di specifiche corrispondenze di immagini ci consiglia di astenerci da raffronti che potrebbero risultar vaghi. Abbiamo preferito attenerci soltanto a quei dati che rendono inoppugnabilesalvo fallace suggestione-la filigrana lopiana nei passi citati. la gelosia è uno dei temi piú ricchi del Cinque e del Seicento e meriterebbe uno studio particolare. Se ne occuparono trattatisti come L. Vives, Varchi, Tasso; creò personaggi della statura di Otello o del Curioso impertinente; $\mathrm{e}$, naturalmente una fungaia di sonetti (inclusi due di Marino), madrigali e componimenti vari; ma, stranamente, nessun emblema o nessuna iconologia, generi ai quali tanto Lope quanto Marino sembrerebbero ispirarsi. Fra i cultori di questo tema Lope merita un posto speciale non solo per i ripetuti trattamenti (“¿Y a quién se debe, Claudio? ¿Y a quién tantas / de celos y de amor definiciones?" poteva vantarsi nell" "Egloga a Claudio," pubblicata postuma ne La Vega del Parnaso ${ }^{4}$ ) ma anche per l'inventività. Marino col fiuto suo sempre finissimo sapeva identificare quanto di nuovo circolava per i sentieri del Parnaso. E sapeva impadronirsene.

Aprofitto di quest'occasione per aggiungere il riscontro di alcune fonti dell'Adone seguendo il metodo già adottato in "Tessere mariniane" che integrano e talvolta risolvono dei dubbi avanzati nello straordinario commento di Giovanni Pozzi

\section{1}

In un carro di palmiti sedere

vedilo altrove, e gir sublime e lieve.

Tirano il carro rapide e leggiere

quattro d'Ircania generose allieve.

Leccano intinto il fren l'orride fere

del bon licor che fa gioir chi'l beve.

Il Pozzi trova il senso di "carro di palmiti" non perspicuo perché "Bacco è incoronato di viti in tutta la tradizione classica; ma è difficile immaginare un carro di pampini. Forse deriva da una lettura distratta di Poliziano, Stanze, 1. 111: 'Vien sopra un carro d'ellera e di pampino/coverto Bacco, il qual duo tigri guidono' (possibile l'equivoco 
causato dall'enjambement)." Ma l'equivoco risale alla lettura di alcuni versi di Stazio:

et iam pampineos matema ad moenia currus promovet, effrenae dextra laevaque secuntur lynces, et uda mero lambunt retinacula tigres

(Theb. IV.656-8)

Lo fa pensare non solo il "pampineos currus" ma anche il particolare delle tigri che leccano le redini molli di vino, particolare assente in Poliziano.

2

Ciò ch'han di molle i morbidi Sabei, gl'Indi fecondi o gli Arabi felici, ciò che produr ne sanno i colli iblei, le piaggie ebalie....

Commentando "piaggie ebalie," il Pozzi: "forse sta per egalie, con scambio possibile nell'orginale forma greca, cioè dell'Acaia: ma non possiamo dire a che cosa alluda." È, però, un'ipotesi nata da evidente distrazione: ebalie è infatti nient'altro che il corrispondente di oebales, cioè "tarantine." Le "piaggie" pugliesi erano note per la loro fertilità (cfr. per es. Virgilio, Geor. IV.125 ss). Si ricordi però che oebalis può significare anche "spartano" o "lacone," da Oebalus, antico re di Sparta, capitale della Laconia ma non mi risulta che questa regione fosse famosa per la sua fertilità o per la produzione di piante aromatiche.

3

spunta mordace il cinnamono altrove

e la pontica noce a piè gli piove

"Mordace cinnamono," secondo il Pozzi, traduce non del tutto propriamente Plinio, Nat. Hist. 12.89: "gignitur in planis quidem, sed densissimis in vepribus rubisque, difficilis collectu." Questo è vero. E l'attributo "nordace" del cinnamono rimarrebbe un hapax se non trovassi un'altra attestazione in un'opera forse nota a Marino: è l'opera di Castore Durante, Herbario nuovo (Roma 1585) dove a p. 130 si legge: "L'eletto cinnamono è quello che è molto odorifero, sottile, e alla lingua mordace."

4

Cosí leon dala mammella irsuta uso ancora a poppar cibi novelli, tosto che l'unghia al piè sente cresciuta, ala bocca le zanne, al collo i velli, già la rupe natia sdegna e rifiuta la tana angusta e le vivande imbelli, 
già segue già tra le cornute squadre per le getule selve il biondo padre.

È similitudine che riprende-in alcuni punti con resa letterale-Stazio:

ut leo, cui parvo mater Caetula cruentos

suggerit ipsa cibos, cum primum crescere sensit

colla iubis torvosque novos respexit ad ungues,

indignatur ali, tandemque effusus apertos

liber amat campos et nescit in antra reverti

(Theb. IX.739-43)

5

Cosí toro non domo a cui le spalle giogo non preme ancor duro e pesante, poiché lasciò nela diletta valle il rival vincitore e trionfante, mugghiando va per solitario calle ...

dipende ancora una volta da Stazio:

...... Veluti dux taurus amata valle carens, pulsum solito quam gramine victor iussit $a b$ erepta longe mugire invenca . . . .

(Theb. II.323-25)

6

E mentre l'aria tepida e molesta move e scaccia il calor noioso e greve, con l'aure vane a vaneggiare intesa sfoga in sospir l'interna fiamma accesa

In questa scena in cui Venere contempla Adone addormentato, bisognava notare una filigrana ricavata dal Parabosco, il primo che, nella Favola d'Adone includa l'elemento del sonno al momento dell'incontro fra i due futuri amanti:

O qualunque tu sia divo o mortale

Che fuggendo il calor noioso e grave. ...

(ott. 10)

7

Quando Venere, travestita da zingara si presenta ad Adone, gli dice:

- Son di Menfi nativa (indi risponde)

barbara donna e per costume errante 
e Adone risponde:

Odo che porta Egitto il primo vanto

dele piú dotte femine presaghe, che d'ogni caso altrui chiaro ed intero san su la mano indovinare il vero

Forse non sarebbe stato superfluo annotare che Menfi era città famosa nell'antichità per il suo numero altissimo di indovine. Ne sono testimoni, tra gli altri, Lucano (Phars., 1.639-41) e Claudiano (De Cons. Mall. Theod. 127).

\section{8}

Come al fischiar del comito supremo, quando ala ciurma incatenata accenna salpar il ferro ed afferrare il remo, stender la vela e sollevar l'antenna, vedesi il legno che con sforzo estremo tosto l'ali per l'acque al volo impenna; freme l'onda percossa, il lito stride, mentre a voga arrancata il mar divide

(XVII.97)

Il Pozzi nota come Claudiano, che Marino in quest'episodio della partenza di Venere da Cipro ha seguito da vicino, non abbia niente di simile. In realtà qui opera il ricordo di un paragone del Poliziano che Marino sottopone a generosa amplificatio:

Come, al fischiar del cómito, sfrenella

La gnuda ciurma e' remi, e mette in voga,

Già per l'aer ne va la schiera snella

(Stanze II.17)

E il paragone polizianesco ricorre in una situazione analoga a quella descritta dal Marino, quando cioè gli Amorini rispondono ad un ordine di Venere.

e Lesbo, che gli accenti estremi udio dela fredda d'Orfeo lingua, circonda

(XVII, 153)

Il Pozzi, commentando sulla "fredda lingua" di Orfeo vede una traduzione libera dell'ovidiano "flebile lingua Murmurat exnimis" (Met. XI.52-53). Ma è una ripresa precisa da Virgilio: "Euridycen vox ipsa et frigida lingua A! miseram Euridycen anima fugiente vocabat" (Geor. IV.525-26).

Ino l'abbraccia e mormorando insieme 
Palemon con Portun rauco ne freme

(XVII.118)

Il Pozzi commentando questa e altre precedenti ottave che elencano nomi di ninfe e divinità marine, ricorda che Melicerte fu trasformato in dio marino che in greco ebbe nome Palemone e in latino Portuno. E aggiunge: "Queste e numerose altre incertezze mostrano come egli [scil. Marino] doveva servirsi di repertori dove, come capita, le notizie sono contaminate e confuse." La diversa identità di Palemone e Portuno risale probabilmente ad Apuleio e precisamente ad un passo che Marino doveva conoscere benissimo visto che si riviene nella storia di Psiche e Cupido: "Adsunt Merei filiolo chorum canentes et Portunus caerulis barbis hispidus et gravis piscoso sinu Salacia et auriga parvulus delphini Palaemon ..." (Met. IV.31).

\section{1}

o come vacca, a cui di sen rapito

abbia il picciol vitel dente inumano

o col maglio crudel rotto e ferito

apiè del sacro altar rigida mano

$(\mathrm{XIX}, 10)$

riprende liberamente negli ultimi due versi Ovidio, Met. II.623-625: "Haud aliter quam cum spectante iuvenca Lactantis vituli dextra libratus ab aure Tempora discussit claro cava malleus ictu."

\section{University of Chicago}

\section{NOTE}

1 "E il tradurre da' Latini e da' Greci si potrebbe pur anche comportare, ma il trasportar gl'interi componimenti di spagnolo in italiano mutando solamente le desinenze, puzza di ladro a piú non posso." Citato da G. Pozzi nella sua ed. dell'Adone 130.

2 Si può cominciare da Fucilla, "Concerning" che riporta ad Ariosto la fonte di un sonetto indicata prima in Lope. Si può continuare con Gasparetti "La 'Galleria' " che per primo indica fonti lopiane nella Galleria e aggiunge un nuovo sonetto al novero dei plagi mariniani e "Ancora un plagio." Vengono poi i numerosi contributi di D. Alonso. E infine, Juan Manuel Rozas.

3 D. Alonso era d'accordo con Fucilla quando s'occupò per la prima volta di quest'episodio: "Lope de Vega." Qualche riserva in Colombo, 24n.

4 Cito dall'ed. di Morby 163-65. Il testo di Lope è dato senza numerazione di versi: essi sono in tutto ottanta (venti quartine) e data la brevità del testo i riscontri sono facili.

5 Il testo di quest'egloga si può vedere nell'ed. Aguilar delle Obras escogidas 2: 398. 


\section{OPERE CONSULTATE}

ALONSO, D. En torno a Lope. Madrid, 1972: 13-108. Rielaborazione di numerosi contributi pubblicati sulle annate 1949-1951 della Revista de filologia espaôla.

FUCILLA, J. "Concerning the Poetry of Lope de Vega." Hispania 15 (1935): 223-42.

GASPARETTI, A. "La 'Galleria' del Cavalier Marino e quella di Dardanio nell' 'Arcadia' di Lope.” Bolitín de la Societad Castellones de cultura 16 (1935): 243-66.

"Ancora un plagio del Marino, ovvero "Padre Zappata predica bene e razzola male." "Boletín de la Societad Castellones de cultura 16 (1935): 327-37.

MENINNI, F. Ritratto del sonetto e della canzone. Napoli, 1677.

ROZAS, J.M. "Lope en la Galería de Marino." Revista de filología española 49 (1966): 91-124. Ristanpato in Sobre Marino y España. Madrid, 1978: 25-67. 